\title{
Procesos de generación de conocimiento y los aspectos que operan en la interacción de actividades colaborativas virtuales en un curso en línea
}

\section{Ana Cecilia Echeverri Echeverri}

Capacitadora profesional de la Universidad Estatal a Distancia de Costa Rica; aecheverri@uned.ac.cr

Recibido: 15 de octubre del 2012
Aceptado: 15 de enero del 2013

\section{RESUMEN}

En este trabajo investigativo se analiza cuáles interacciones didácticas y mecanismos de apoyo docente, producidos en una situación de aprendizaje colaborativo, contribuyen a la gestión de nuevo conocimiento personal y colectivo Para ello, se realizó un análisis de dos espacios colaborativos en el curso virtual Evaluación de programas y proyectos en Educación a Distancia, que forman parte del plan de estudios de la Maestría en Educación a Distancia de la Universidad Estatal a Distancia de Costa Rica, UNED. En el análisis de las interacciones se utilizó el modelo propuesto por Garrison Anderson y Archer que permite determinar los aspectos que contribuyen a la construcción de aprendizaje significativos en un entorno de aprendizaje colaborativo.

\section{PALABRAS CLAVE}

Interacción, aprendizaje colaborativo, gestión de conocimiento, aprendizaje virtual, apoyo didáctico.

\section{SUMMARY}

In this research paper which discusses educational interactions and teacher supportmechanisms, produced in a collaborative learning situation, contribute to the management of personal and collective new knowledge To do this, an analysis of two collaborative spaces in the virtual course evaluation programs and projects in Distance Education, part of the curriculum of the Master of Distance Education of the State Distance University of Costa Rica, UNED. In the analysis of the interactions we used the model proposed by Garrison Anderson and Archer which determines the aspects that contribute to the construction of significant learning in a collaborative learning environment.

\section{KEY WORDS}

Interaction, collaborative learning, knowledge management, virtual learning, instructional support.

\section{INTRODUCCIÓN}

El aprendizaje a partir de la experiencia es una característica del ser humano. Pero, además de esta realidad se debe procurar que el conocimiento, construido en el proceso formativo, sea significativo para la persona, o sea, que tenga sentido en la cotidianidad. En la búsqueda de la comprensión y significancia del aprendizaje, se han planteado desde diferentes corrientes educativas el uso de diversas estrategias y recursos. De manera particular, se destacan las posibilidades que herramientas como el Internet y los nuevos planteamientos teóricos, alrededor de la realidad que imprimen a los procesos de aprendizaje. 
Paralelo al desarrollo de la sociedad del conocimiento se han generado las redes de aprendizaje, como un medio para el trabajo colaborativo y la formación continua. Las redes de aprendizaje se han constituido como una característica del nuevo modo de creación del conocimiento. De manera especial, las redes apoyadas en las Tecnologías de la Información y la Comunicación (TIC) se han convertido en una herramienta eficaz para el aprendizaje colaborativo; según Castells (1996), la comunicación en red interactiva es una transformación tecnológica de gran dimensión, después de la invención del alfabeto.

En este marco, es necesario reconocer las características y actividades de las estrategias de aprendizaje, que favorezcan la gestión del conocimiento con sentido, pertinencia y con interacción social. Para encontrar un contexto bajo estas condiciones se requiere de la discusión profunda sobre los pilares epistemológicos, ontológicos y axiológicos en los cuales se fundamenta cualquier propuesta educativa.

\section{- La gestión del conocimiento}

El proceso de aprendizaje, como objeto de estudio, ha sido discutido desde diversas disciplinas y desde diferentes enfoques del conocimiento. Para López y Gallego (2005), la gestión del conocimiento es la actividad de "creación, obtención, organización, clasificación, integración y distribución del conocimiento" (p. 32).

Para hablar de cómo se conoce el objeto, o sea, cómo se asume la realidad, se debe reconocer la visión o planteamiento teórico que permite explicar el camino que se recorre para alcanzar dicho conocimiento. Este proceso requiere identificar la visión desde donde se asume, para Khum (1962) un paradigma se debe entender como una comunidad de partidarios en la actividad científica, diferenciada de otras posturas científicas, con la característica de permitirse todo tipo de problemas por resolver, no obstante, las explicaciones científicas sobre el proceso de construcción del conocimiento implican también, según Maturana (1990), explicar al ser humano, a la persona que conoce bajo las circunstancias contextuales de su reflexión. Lo anterior exige, igualmente, una convivencia en el lenguaje y en la experiencia común, para entender lo expresado y conocido por parte del aprehendiente.

\section{- La cognición distribuida y el aprendizaje situado}

El aprendizaje, en el sentido expuesto en esta investigación, implica concebir su proceso como un todo, donde actores, medios, recursos y procesos se combinan en una dinámica compleja, total y en estrecha relación con su entorno social. Para Bohm (1998), las nuevas formas de reconocer y relacionarnos con el mundo implican "descubrir por nosotros mismos el significado de la totalidad" (p. 50).

Algunas teorías del aprendizaje ubican la cognición de la persona, de manera individual, como el aspecto fundamental para alcanzar el conocimiento sobre los objetos y la naturaleza misma. Pea (1993) y Sangè (1995) señalan que la inteligencia producida en equipo funciona sobre las relaciones y fines que se alcanzan a través de las realizaciones, y no sobre la mente de cada persona.

Desde la teoría social vigotskiana se plantea que en los procesos de aprendizaje las condiciones históricas, culturales y sociales ejercen una influencia sobre el desarrollo individual, en donde según Daniels (2003): "una posible extensión de este punto de partida es proponer que todo pensamiento está inextricablemente ligado al contexto" (p. 65)

Las Tecnologías de la Información y la Comunicación, y los nuevos estudios sobre el funcionamiento del cerebro, han permitido evidenciar que conocemos en interacción y bajo un contexto particular. Para Pea (1993): "El que observa de cerca las prácticas cognitivas tiene la 
impresión de que la "mente" raramente trabaja sola. Las inteligencias reveladas mediante esas prácticas están distribuidas en la mente y entre las personas, y los entornos simbólicos y físicos, tanto naturales cuanto artificiales". (p. 75).

\section{Redes de aprendizaje y el trabajo colaborativo}

Fernández (2008) sugiere que técnicamente una red "es un conjunto de nodos (o individuos) y de vínculos (o relaciones) entre ellos" (p. 12), y en una comparación con las estructuras de equipos y organizaciones destaca las siguientes características: tienen vínculos más débiles, sus fronteras son más permeables y el sentido de solidaridad es más especializado y cambiante.

La metáfora de la telaraña utilizada en el contexto de las redes ha permitido comprender esta nueva forma de organización, que Chapman (2008) define como un "reticulado global -un nexo cognitivo unificado y unificador- de la teoría que tenemos sobre el mundo, las formas de conocerlo y de pensar en él" ( $p$. 21). Desde esta visión, las redes de aprendizaje se constituyen a partir de la identificación de postulados epistemológicos y ontológicos para convertirse en un espacio de aprendizaje significativo y contextual.

Como características de las redes de conocimiento se destacan las propuestas por Harasim (2000), en el sentido de que se basan en el aprendizaje autodirigido, no hay temario prescrito, se produce en la interacción y se dispone de una variedad de materiales, la calidad de la interacción y la motivación aumentan, es un espacio que incluye a personas con discapacidades, estimulan el aprendizaje colaborativo.

El trabajo colaborativo como estrategia se inscribe dentro de un enfoque constructivista del aprendizaje. Como metodología se centra en las interacciones y en el aporte de las personas que participan en los procesos de construcción de conocimiento desde una visión integral y holística.

Para comprender las características y procesos que se dan en el aprendizaje colaborativo es necesario explicitar desde cual visión de aprendizaje partimos. Para Laszlo (1995) el aprendizaje es un proceso de creación de redes, donde los procesos mentales se comportan como una red interna neuronal, en la que conectamos y moldeamos información y fuentes de conocimiento. Los seres humanos las creamos en cada momento y de forma continua para adquirir, experimentar, crear y conectar nuevos conocimientos.

\section{- El pensamiento holográfico y el aprendizaje}

Para Wilber (1992): "Una de las propiedades misteriosas del holograma y del cerebro estriba en la distribución de información a través del sistema con cada fragmento codificado para producir información del todo" (p. 16). Visión a partir de la cual se puede intuir que el cerebro no es un conjunto de partes, donde se desarrollan de manera aislada las funciones. Igualmente, desde la biopedagogía se plantea que el ser humano en su capacidad vital, es un sistema determinado estructuralmente y con una organización propia, que llega a constituirse como tal, desde las interacciones en su medio, (Maturana, 2003). Son estos avances, desde las diferentes ciencias, lo que le dan fundamentación a las estrategias colaborativas en los procesos de formación y a las redes como el espacio idóneo para promover aprendizajes significativos.

Por lo anterior, diseñar propuestas pedagógicas interdisciplinarias, que según Morín (2003) deben verse como la ecología de las ideas y la acción de todos los participantes en el proceso, es un aspecto importante para construir aprendizajes significativos, tanto para participantes como para la comunidad, que se genera en la vivencia de los espacios de aprendizaje. 


\section{- La interacción didáctica}

En los procesos de aprendizaje en red, la comunicación es parte esencial del proceso mismo, es el vehículo a través del cual el conocimiento local, de cada persona, se traslapa y permite la generación de nuevo conocimiento, del conocimiento explícito personal y del colectivo.

Las relaciones de interacción en una comunidad de aprendizaje en red emergen de la dinámica propia y de los propósitos que permitieron su confirmación como grupo. Para Sangrè (1997) en el trabajo de equipo las personas tienen cambios en sus aptitudes y en la forma de percibir la realidad, a partir de los cuales se alcanzan nuevos aprendizajes.

En este mismo sentido, Maturana y Varela (1984) se refieren a la comunicación como la interacción multidimensional que se da a través de diferentes espacios, físico, biológico, psicológico, donde "el fenómeno de la comunicación no depende de lo que se entrega, sino de lo que pasa con lo que se recibe" (p. 16o), aspecto diferente de transmitir información.

La comunicación, en su sentido más general, se concibe como el medio que permite el intercambio de expresiones comunicativas a través del lenguaje; elemento que en el acto educativo se convierte en el soporte de las interacciones que permiten el flujo de información y emociones. Para Robert (s.f.)

El lenguaje humano nace de y hace posible el proceso de aprendizaje. Esto nos permite pensar que comunicación, lenguaje y aprendizaje se retroalimentan mutuamente en bucles y que la información, las conductas y los compromisos que se generan tienen una influencia activa sobre ambos procesos (p. 26)

De manera particular, la comunicación en red ofrece a los participantes de las diferentes comunidades de aprendizaje un espacio para la generación del conocimiento, como claramente lo expresa Cabero (2008): "los sistemas informáticos basados en tecnologías de creación de redes semánticas... son los que van a adquirir preponderancia en el futuro" (p. 97).
Para Harasim et al (2000), en las redes de aprendizaje se establece una comunicación de conocimientos basada en el "uso de vínculos electrónicos entre distintas comunidades de profesores y estudiantes para facilitar la adquisición de información y de conocimientos" ( $p$. 31), donde lo importante es el objetivo de interactuar y hacer uso de la información que se necesita para la construcción del conocimiento.

\section{- Los recursos tecnológicos y la interacción virtual}

La interacción en la educación a distancia ha estado mediada por diferentes recursos y a través del tiempo han evolucionado hasta llegar a las tecnologías digitales que hoy se utilizan en los diferentes procesos educativos. Según Prieto y Van de Pol (2006), el valor pedagógico de las tecnologías digitales "le viene de su mediación para promover y acompañar el aprendizaje" (p. 134), no siendo el recurso en sí mismo lo central de la propuesta pedagógica.

Para Vilaseca y Meseguer (2006), en la educación virtual es básico que se provea un entorno que facilite tanto la interacción personal como la interacción con los contenidos, para alcanzar un proceso de aprendizaje de calidad y con sentido para el o la estudiante. La interacción en los entornos en línea se desarrolla a través de los diferentes recursos didácticos, propios de los sistemas virtuales de aprendizajes, denominados también sistema de gestión, como Blackboard y Moodle, plataformas utilizadas en la UNED.

Uno de los recursos más utilizados para la interacción en los cursos virtuales son los foros de discusión. La participación en los foros debe analizarse a través de diferentes aspectos. Henri (1992), citado por Gairín y Muñoz diferenció entre dimensiones participativas e interactivas, para referirse a la calidad de las participaciones y su aporte a la construcción de conocimiento, afirmando que la cantidad de participaciones no es un indicador válido. 
En este mismo sentido, Garrison, citado por Fallas (2008) afirma que

El modelo de investigación práctica ofrece un medio para juzgar la naturaleza y calidad de la reflexión y el discurso crítico en una comunidad de investigación que trabaja en cooperación, y donde se utilizan indicadores para evaluar el nivel latente y patente de pensamiento y discurso críticos en el número de mensajes que se generan durante las sesiones virtuales (p. 4).

Desde los diferentes modelos investigativos, la relación entre las interacciones producidas en los foros de discusión y la construcción de aprendizaje significativo, depende de varios factores. La reflexión crítica de cada participante sobre la realidad estudiada, las posibilidades del contexto individual y colectivo, junto con la propuesta pedagógica sugerida para el acto educativo son aspectos que contribuyen a la construcción social.

\section{- Características del curso analizado}

La naturaleza del curso es teórico-metodológi$\mathrm{ca}$, donde el estudiante reconozca los diferentes aspectos teóricos que intervienen en la evaluación de los programas y proyectos que se desarrollen en la Educación a Distancia, como calidad, técnicas y metodologías, estándares internacionales y nacionales para la acreditación de la educación superior.

Los contenidos y el desarrollo de la propuesta curricular se hizo en tres módulos y bajo tres dimensiones: cognitiva (saber conocer), procedimental (saber hacer) y actitudinal (saber ser). El curso estaba orientado a alcanzar las competencias mínimas en la valoración, análisis y evaluación de programas y proyectos que se ejecuten en la Educación a Distancia.

La metodología utilizada en la mediación pedagógica, para la generación del aprendizaje en el curso analizado, incluyó la pregunta generadora, actividades de comprensión y elaboración de propuestas, socialización de aprendizajes, la autoevaluación y coevaluación. El cuadro N. ${ }^{\circ}$ 1 describe las estrategias de mediación utilizadas en el curso.

En el curso participaron diez estudiantes: seis mujeres y cuatro hombres. En su mayoría funcionarios(as) de la misma universidad, que buscan la formación profesional en programas y proyectos educativos a distancia; aspecto que permite la contextualización de los contenidos y estrategias de aprendizaje sugeridas en la propuesta pedagógica.

Cuadro 1. Estrategias de mediación utilizadas en el curso Evaluación de programas y proyectos de educación a distancia

\begin{tabular}{|l|l|}
\hline \multicolumn{1}{|c|}{ Estrategia } & \multicolumn{1}{c|}{ Proceso cognitivo } \\
\hline Foro de conocimiento. & Evidencia de conocimiento. \\
\hline Cuestionario de preguntas abiertas. & Evidencia de conocimiento base. \\
\hline Prueba de competencia. & Evidencia de competencia cognitiva. \\
\hline Trabajo colaborativo. & Evidencia de producto. \\
\hline Foro de circunstancia. & Evidencia de conocimiento circunstancial. \\
\hline
\end{tabular}

Fuente: Arce y Rojas (2009) 


\section{METODOLOGÍA}

El presente trabajo investigativo tuvo un enfoque cualitativo y una visión sistémica y dinámica de las relaciones y elementos implícitos en el contexto, para analizar las diferentes situaciones y la multiplicidad de aspectos que componen el objeto de estudio, la interacción y la mediación, para interpretar la dinámica de los procesos que se dan en la gestión del conocimiento

El marco práctico del proyecto investigativo expone los elementos constitutivos del curso Evaluación de programas y proyectos en
Educación a Distancia, que forman parte del plan de estudios de la Maestría en Educación a Distancia de la Universidad Estatal a Distancia de Costa Rica, UNED. El curso y sus participantes son, a la vez, la muestra general y el objeto de la investigación; dos casos que se observarán en el proceso de evaluación, un foro temático general y un foro grupal. A continuación se describen las categorías utilizadas y las fuentes de análisis.

El análisis de los datos cualitativos se ha realizado con apoyo del software de análisis Atlas.ti, este se sustenta en la Teoría Fundamentada sustentada

Cuadro 2. Categorías y recursos de análisis

\begin{tabular}{|c|c|c|c|c|}
\hline Categoría & & & & \\
\hline Recurso de análisis & Análisis documental & Participantes & Apoyo docente & Investigadora \\
\hline $\begin{array}{l}\text { 1. Mecanismos de } \\
\text { apoyo docente para } \\
\text { el aprendizaje cola- } \\
\text { borativo. }\end{array}$ & $\begin{array}{l}\text { 1.1 Programa del } \\
\text { curso. } \\
\text { 1.2 Marco teórico. }\end{array}$ & $\begin{array}{l}\text { 1.1 Participación en } \\
\text { el foro temático. }\end{array}$ & $\begin{array}{l}\text { 1.1 Programa del } \\
\text { curso. } \\
\text { 1.2 Entrevista a } \\
\text { facilitadora. } \\
\text { 1.3 Foro Temático. }\end{array}$ & $\begin{array}{l}\text { 1.1 Observación } \\
\text { participante en } \\
\text { el foro temático } \\
\text { general. }\end{array}$ \\
\hline $\begin{array}{l}\text { 2. Estrategias de } \\
\text { aprendizaje colabo- } \\
\text { rativo. }\end{array}$ & $\begin{array}{l}\text { 2.1 Programa del } \\
\text { curso. } \\
\text { 2.2 Marco metodo- } \\
\text { lógico. }\end{array}$ & $\begin{array}{l}\text { 2.1 Propuestas de } \\
\text { trabajo en grupo. }\end{array}$ & $\begin{array}{l}\text { 2.1 Programa del } \\
\text { curso } \\
\text { 2.2 Actividades } \\
\text { propuestas en el } \\
\text { curso. } \\
\text { 2.3 Preguntas gene- } \\
\text { radoras. }\end{array}$ & $\begin{array}{l}2.1 \text { Análisis del foro } \\
\text { temático general. } \\
\text { 2.2 Análisis del foro } \\
\text { grupal. }\end{array}$ \\
\hline $\begin{array}{l}\text { 3. Interacciones } \\
\text { didácticas. }\end{array}$ & $\begin{array}{l}\text { 3.1 Foro temático. } \\
\text { 3.2 Foro de grupo. }\end{array}$ & $\begin{array}{l}3.1 \text { Interacciones } \\
\text { entre pares. }\end{array}$ & $\begin{array}{l}\text { 3.1. Interacción } \\
\text { tutora-estudiante. }\end{array}$ & $\begin{array}{l}3.1 \text { Observación } \\
\text { participante en los } \\
\text { foros. }\end{array}$ \\
\hline $\begin{array}{l}\text { 4. Actitudes que } \\
\text { facilitan el trabajo } \\
\text { colaborativo. }\end{array}$ & $\begin{array}{l}\text { 4.1 Programa del } \\
\text { curso. } \\
\text { 4.2 Marco practico. }\end{array}$ & $\begin{array}{l}\text { 4.1 Perfil para el } \\
\text { trabajo colaborativo } \\
\text { (Escala de likert). } \\
\text { 4.2 Dinámica de } \\
\text { trabajo en el foro } \\
\text { temático. } \\
\text { 4.3 Marco teórico. }\end{array}$ & $\begin{array}{l}\text { 4.1 Dinámicas de } \\
\text { trabajo sugeridas. } \\
4.2 \text { el diseño de las } \\
\text { actividades en el } \\
\text { programa del curso. } \\
4.3 \text { Entrevista a } \\
\text { facilitadora. }\end{array}$ & $\begin{array}{l}\text { 4.1 Análisis de la } \\
\text { escala. }\end{array}$ \\
\hline $\begin{array}{l}\text { 5. Recursos colabo- } \\
\text { rativos de la platafor- } \\
\text { ma virtual. }\end{array}$ & $\begin{array}{l}\text { 5.1 Recursos de } \\
\text { aprendizaje propues- } \\
\text { tos en el programa. }\end{array}$ & $\begin{array}{l}5.1 \text { Utilización de los } \\
\text { recursos. }\end{array}$ & $\begin{array}{l}5.1 \text { Estrategias de } \\
\text { trabajo sugeridas. }\end{array}$ & $\begin{array}{l}5.1 \text { Análisis de los } \\
\text { recursos que contie- } \\
\text { ne la plataforma. }\end{array}$ \\
\hline
\end{tabular}

Fuente: Echeverri (2011) 
por Glaser y Strauss, que plantean la necesidad del rigor en el manejo de los datos para el análisis en la investigación cualitativa. Este recurso permite a partir de la recolección y organización de la información obtenida categorizar y codificar como un primer nivel y el establecimiento de relaciones significativas para la exploración de las categorías de análisis definidas previamente y las categorías emergentes durante el proceso de análisis, como un segundo nivel.

\section{RESULTADOS}

Para encontrar la relación que existe entre los procesos de generación de conocimiento y los aspectos que operan en la interacción de actividades colaborativas virtuales, se analizaron los elementos de una propuesta pedagógica que favorecen el aprendizaje pertinente y significativo para todos los y las estudiantes.

Un primer elemento que se analizó fue la propuesta pedagógica del curso para alcanzar los objetivos de aprendizaje. El Cuadro 3 muestra la organización de la secuencia de actividades que se desarrollaron en el curso.

Un segundo aspecto analizado tiene que ver con la propuesta de investigación práctica de Garrison que identifica cuatro fases en la detección de la presencia de pensamiento reflexivo y crítico, que son la base para un aprendizaje significativo. Para el análisis, este modelo en esta investigación se complementa con otros autores que trabajan la generación de conocimiento. El pensamiento crítico que se genera en una actividad de aprendizaje colaborativo, se entiende como un proceso y un resultado a la vez, siendo este último de dimensión individual. En el Cuadro 4 se presentan los descriptores, indicadores y evidencias para el caso estudiado en esta investigación.

En la estrategia Foro temático, de un total de 57 participaciones se desglosan como se muestra en el Cuadro 5.

Cuadro 3. Construcción colaborativa de la propuesta de evaluación en el curso Evaluación de programas y proyectos en educación a distancia

\begin{tabular}{|c|c|}
\hline \multicolumn{2}{|c|}{$\begin{array}{l}\text { Foro grupal } \\
\begin{array}{l}\text { Cada grupo utilizó la herramienta foro para construir el diseño de evaluación de su programa } \\
\text { o proyecto, para ello se realizaran las siguientes actividades }\end{array}\end{array}$} \\
\hline Organización de la propuesta & Diseñar la propuesta de evaluación de proyecto o programa. \\
\hline Ensayo & $\begin{array}{l}\text { Realizar la justificación de la propuesta evaluativa a partir de } \\
\text { preguntas generadoras. }\end{array}$ \\
\hline Comparación de modelos & Comparar los modelos de evaluación utilizados en tres países. \\
\hline Avance de proyecto II & Construir el marco conceptual de la propuesta evaluativa. \\
\hline Avance de proyecto III & $\begin{array}{l}\text { Construir el marco metodológico de la propuesta evaluativa y } \\
\text { presentar el informe final. }\end{array}$ \\
\hline Socialización de propuesta & $\begin{array}{l}\text { Incorporar las observaciones realizadas por los pares y perso- } \\
\text { nal docente. }\end{array}$ \\
\hline
\end{tabular}


Cuadro 4. Fases del modelo de investigación práctica de Garrison

\begin{tabular}{|l|l|l|l|}
\hline \multicolumn{1}{|c|}{ Fase } & \multicolumn{1}{|c|}{ Descriptor } & \multicolumn{1}{c|}{ Indicador } & \multicolumn{1}{c|}{ Evidencia } \\
\hline $\begin{array}{l}\text { Hecho } \\
\text { desencadenante }\end{array}$ & Inductivo & $\begin{array}{l}\text { Reconocimiento del contexto del } \\
\text { problema }\end{array}$ & $\begin{array}{l}\text { Aclaración del problema y su } \\
\text { contexto de aplicación. }\end{array}$ \\
\hline $\begin{array}{l}\text { Exploración } \\
\text { de ideas }\end{array}$ & Exploratorio & $\begin{array}{l}\text { Análisis del problema a través de } \\
\text { diferentes recursos }\end{array}$ & $\begin{array}{l}\text { Búsqueda de la información clave } \\
\text { para la resolución del problema. }\end{array}$ \\
\hline Integración & Integrador & $\begin{array}{l}\text { Síntesis de la reflexión para encon- } \\
\text { trar los puntos de convergencia }\end{array}$ & $\begin{array}{l}\text { Aproximación de la propuesta } \\
\text { resolutoria. }\end{array}$ \\
\hline Resolución & Deductivo & $\begin{array}{l}\text { Construcción de propuestas al pro- } \\
\text { blema planteado }\end{array}$ & $\begin{array}{l}\text { Valoración colaborativa de la so- } \\
\text { lución del problema, se requiere } \\
\text { presentar resultados. }\end{array}$ \\
\hline
\end{tabular}

Cuadro 5. Interacciones en foro temático general del curso Evaluación de programas y proyectos en educación a distancia

\begin{tabular}{|c|c|c|c|}
\hline Fase & Participaciones & Porcentaje & Evidencia \\
\hline $\begin{array}{l}\text { Hecho } \\
\text { desencadenante }\end{array}$ & 13 & $23 \%$ & $\begin{array}{l}\text { "De ahí que, se debe entender la evaluación como } \\
\text { el aspecto que permite, de manera dinámica, el } \\
\text { cambio constante de los elementos que intervie- } \\
\text { nen en un proyecto para alcanzar la excelencia". }\end{array}$ \\
\hline $\begin{array}{l}\text { Exploración } \\
\text { de ideas }\end{array}$ & 23 & $40 \%$ & $\begin{array}{l}\text { "El evaluador requiere de algunas competencias y } \\
\text { una de ellas es la criticidad, la presencia de ella le } \\
\text { ayudará a valorar, analizar y concretar los aciertos } \\
\text { y los errores en que se ha incurrido ante el diseño } \\
\text { e implementación de un programa o proyecto". }\end{array}$ \\
\hline Integración & 12 & $21 \%$ & $\begin{array}{l}\text { "Maribel y José Luis: enfocan dos aspectos fun- } \\
\text { damentales del proceso evaluativo, su papel en la } \\
\text { planificación, ejecución y finalización de un pro- } \\
\text { yecto (recordemos que tienen un plazo), si bien lo } \\
\text { podemos visualizar como un eje trasversal, tam- } \\
\text { bién podemos valorarlo como un componente } \\
\text { más del proceso evaluativo". }\end{array}$ \\
\hline Resolución & 9 & $16 \%$ & $\begin{array}{l}\text { "Nos hemos referido al concepto en sí de la eva- } \\
\text { luación, de sus finalidades, de su proceso, de los } \\
\text { elementos que intervienen en ella, de sus carac- } \\
\text { terísticas, del papel del evaluador y de manera } \\
\text { específica, creo que lo referente a los criterios o } \\
\text { indicadores que enmarcan el proceso de evalua- } \\
\text { ción es un aspecto muy importante". }\end{array}$ \\
\hline Total & 57 & $100 \%$ & \\
\hline
\end{tabular}




\section{CONCLUSIONES}

Los procesos de aprendizaje colaborativos son espacios idóneos para una mediación pedagógica desde el aprendizaje, donde el auto y el interaprendizaje se conjugan para crear las condiciones idóneas para la generación de conocimientos. Mediar en la comprensión del objeto de estudio, en la regulación de los aprendizajes y en la consecución de conocimientos significativos adquiere una nueva dimensión en los espacios de aprendizaje en red, por ejemplo, en el foro de trabajo grupal la participación individual fue decisiva para la producción colectiva de los aprendizajes, determinada por la propuesta pedagógica de construcción del producto final, trabajado a partir de una dinámica colaborativa.

De manera particular, el modelo de investigación de Garrison y Anderson complementado con los planteamientos de otros autores, permitió evidenciar que la presencia docente, representada en la propuesta integral del curso y en la facilitación del mismo es esencial para generar reflexión crítica en los foros virtuales de aprendizaje. La participación pertinente de la docente del curso en cada una de las fases del modelo a través de una comunicación constante, el aporte a la búsqueda de información clave y propuesta de solución a partir del trabajo colaborativo fueron aspectos relevantes en la generación de aprendizaje en los diferentes espacios de aprendizaje del curso.

Igualmente, en la propuesta metodológica del curso analizado, todas las actividades de aprendizajes sugeridas evidencian que la intencionalidad de la propuesta de formación era propiciar espacios para el aprendizaje autónomo e independiente. Donde la metodología estaba centrada en la producción de interacciones relevantes y desde la construcción colaborativa del aprendizaje.

De manera particular, la investigadora encontró que la interdisciplinariedad, que se dio en el grupo analizado jugó un papel muy importante en la generación de nuevos aprendizajes. Los aportes de cada participante desde sus dominios conceptuales y vivenciales fueron determinantes para la calidad y profundidad de cada uno de las actividades de aprendizaje y evaluación promovidas en el curso.

\section{REFERENCIAS}

Bohm, David. (1988). La totalidad y el orden implicado. España: Editorial Kairós S.A.

Cabero, Julio y Pedro Román. (2005). E-actividades: un referendo básico para la formación en Internet. España: Eduforma.

Castells, M. y otros. (1997). Nuevas perspectivas críticas en educación. España: Ediciones Paidós Ibérica, S.A.

Castells, M. (1996). La era de la información: Economía, sociedad y cultura. Capítulo 5. España: Alianza Editorial.

Chapman, Judith, (2008). ¿Por qué redes y por qué ahora? Cuadernos de pedagogía. No. 385, 20-23.

Daniels, H. (2003). Vigotsky y la pedagogía. España: Editorial Paidós

Fallas, I. (s.f.). ¿Está mi foro produciendo pensamiento crítico?. Ponencia al Ponencia presentada en el XIV Congreso Internacional de Tecnología y Educación a Distancia. San José, Costa Rica, del 5 al 7 de noviembre de 2008. Tomado desde http:// www.uned.ac.cr/XIVCongreso/

Fernández, Mariano. (2008). ¿Qué hay de nuevo bajo el sol?. Cuadernos de pedagogía. No. 385, 12-19.

Garrison, D. y otros. (2004). Critical Thinking, Cognitive Presence, and Computer Conferencing in Distance Education. Extraído el 22 de abril del 2010 desde http://communityofinquiry.com/ files/CogPres_Final.pdf

Harasim, L. y otros. (2000). Redes de aprendizaje: guía para la enseñanza y el aprendizaje en red. España: Gedisa Editorial

Kuhn, T. (1962). La estructura de las revoluciones científicas. México: Fondo de cultura económica. 
López, P. y D. Gallego (2005). Propuesta de un ciclo de vida para creación y gestión del conocimiento. Actualización del análisis de las funcionalidades de las aplicaciones informáticas para la gestión de conocimiento. RIED v. 8: 1 y 2, 2005, pp 31-66

Maturana, H. (1999). Transformación en la convivencia. Venezuela: Dolmen Ediciones.

Maturana, H y F. Varela. (1984). El árbol del conocimiento. Santiago, Chile: Editorial Universitaria.

Morín, E. 2003. El Método V: La humanidad de la humanidad. Ediciones Cátedra: Madrid.

Prieto, D. y P. van de Pol. (2006). E-learning: comunicación y educación, el diálogo continua en el ciberspacio. Colombia: RNTC
Robert, A. (2009). La biopedagogía. Tomado desde http://issuu.com/irenecr/docs/name306e34

Sangrè, P. y otros. (1997). La quinta disciplina en la práctica, cómo construir una organización inteligente. España: Industria Gráfica Bigsa

Vilaseca, Jordi y otros. (2006). E-learning, competencias y micronización de contenidos en economía y empresa. Tomado desde http://www. iadis.net/dl/final_uploads/200607C048.pdf

Wilber, k. y otros. (1992). El paradigma holográfico: una exploración en las fronteras de la ciencia. España: Editorial Kairós 